\title{
Erasure Insertion in RS-Coded SFH MFSK Subjected to Tone Jamming and Rayleigh Fading
}

\author{
Sohail Ahmed, Lie-Liang Yang and Lajos Hanzo \\ School of ECS, University of Southampton, SO17 1BJ, UK. \\ Tel: +44-23-8059 3364, Fax: +44-23-8059 4508 \\ Email: sa03r@ecs.soton.ac.uk;lly@ecs.soton.ac.uk; lh@ecs.soton.ac.uk; http://www-mobile.ecs.soton.ac.uk
}

\begin{abstract}
The achievable performance of Reed Solomon (RS) coded slow frequency hopping (SFH) assisted $M$-ary frequency shift keying (MFSK) using various erasure insertion (EI) schemes is investigated, when communicating over uncorrelated Rayleigh fading channels in the presence of multitone jamming. Three different EI schemes are considered, which are based on the output threshold test (OTT), on the ratio threshold test (RTT) and on the joint maximum output-ratio threshold test (MO-RTT). The relevant statistics of these EI schemes are investigated mathematically and based on these statistics, their performance is evaluated in the context of error-and-erasure RS decoding. It is demonstrated that the system performance can be significantly improved by using error-and-erasure decoding invoking the EI schemes considered.
\end{abstract}

\section{INTRODUCTION}

In slow frequency hopping (SFH) assisted $M$-ary frequency shift keying (MFSK) using Reed Solomon (RS) coding, typically errorand-erasure decoding is employed for enhancing the achievable system performance when encountering interference and/or jamming. Erasure insertion (EI) implies that an RS-coded MFSK symbol, which is deemed to be unreliable owing to fading, interference or both is erased. EI enhances the error correcting capability of the RS decoder. Various EI schemes designed for supporting error-anderasure decoding in conjunction with SFH-MFSK receivers have been proposed in the open literature [1]-[3]. In this paper, we complement the results of [3] by investigating the performance of RS-coded SFH-MFSK system when communicating over uncorrelated Rayleigh fading channels in the presence of tone jamming. In our analysis three different types of low complexity EI schemes are considered, which include the output threshold test (OTT), the ratio threshold test (RTT) and the joint maximum output-ratio threshold test (MO-RTT), which were considered in [3]. The decision statistics associated with these EI schemes are analyzed employing an approach similar to that used in [3]. With the aid of these decision statistics, we derive analytical expressions for the RS codeword error probability and the bit error rate (BER) after erasure insertion. We will demonstrate that these EI schemes are capable of providing useful information for improving the performance of error-and-erasure decoding. The remainder of this paper is structured as follows. In Section II the system under consideration is described and the symbol error rate of the uncoded system is derived. In Section III the EI schemes considered are discussed and the related decision statistics are investigated, while in Section IV the numerical results are presented. Finally, in Section V we present our conclusions.

The financial support of the the EPSRC, UK and EU under the auspices of the Phoenix and Newcom projects, as well as that of the Government of Pakistan is gratefully acknowledged.

\section{SYSTEM DESCRIPTION}

The system under consideration is similar to that described in [3] and [4]. In the transmitter, the RS encoder of code rate $R_{c}=k / n$ converts each block of $k M$-ary information symbols into $n$ coded symbols. We assume that we have $n=M-1=2^{b}-1$ where $b=\log _{2} M$ represents the number of bits per information symbol. After MFSK modulation the frequency synthesizer, which operates under the control of a pseudo noise (PN) generator, generates a sequence of random hopping frequencies, one of which is activated during each hop interval of duration $T_{h}$ or symbol interval $T_{s}$, where we assume that $T_{h}=T_{s}$. We also assume that the bandwidth of one frequency hopping $(\mathrm{FH})$ tone is given by that of its main spectral lobe occupying $B=1 / T_{h}$.

The modulated signal of each FH tone is transmitted over a frequency non-selective fading channel obeying Rayleigh distribution. We assume that the fading is sufficiently slow so that its amplitude and phase remain constant within a symbol duration and that fading is independent for each symbol, which may be achieved with the aid of interleaving. Consequently, when assuming Rayleigh fading, the probability density function (PDF) of the amplitude attentuation factor is given by [5]

$$
p_{\alpha}(r)=\frac{2 r}{\Omega} e^{-r^{2} / \Omega}, \quad r \geq 0
$$

where $\Omega=E\left[\alpha^{2}\right]$.

The transmitted signal is assumed to be interferred by a tone jamming signal consisting of $Q$ equal-power continuous wave tones, each of which has a frequency equal to that of one of the $M$-ary signalling tones. We consider the case of $n=1$-band multitone jamming (MTJ), which is regarded as the worst case tone jamming scenario [6]. In this context, the intentional jammer distributes the interfering tones in such a way that there is at most one interfering tone in an MFSK band, defined by the bandwidth occupied by the $M$ FSK signalling tones. Furthermore, it has been shown that for Rayleigh fading a jammer associated with a jamming duty factor of unity inflicts the most detrimental interference upon the FH system [7]. These assumptions imply that we have $Q=N$, where $N$ is the number of MFSK bands in the total spread spectrum bandwidth given by $W_{s s}=M \times N \times B$, which is assumed to be fixed. We assume that the intentional jammer has explicit knowledge of the communication system's parameters and thus it is capable of adjusting the number of jamming tones accordingly, keeping the total MTJ power fixed. We can define the Signal to Jammer Power Ratio (SJR) as [6]

$$
S J R=\frac{E_{b}}{P_{T J} / W_{s s}}=\frac{E_{b}}{P_{J}} \frac{N}{Q} \frac{M}{T_{s}}=\frac{E_{s}}{E_{j}} \frac{N}{Q} \frac{M}{b},
$$

where $E_{s}=P T_{s}=b E_{b}$ represents the symbol energy, $E_{b}$ is the energy per bit and $P$ is the power of the transmitted signal. 
Furthermore, in (2) $P_{T J}=P_{J} Q$ represents the total MTJ power, $P_{J}$ is the power of a single MTJ tone and $E_{j}$ represents the energy of a jamming tone per symbol duration.

In the receiver, the signal is de-hopped and then demodulated by a bank of $M$ square-law detectors. An EI device then observes all the $M$ square-law detector outputs and either inserts an erasure, if the corresponding threshold condition is met (to be discussed in Section III), or outputs a symbol according to the standard MFSK demodulation principle. After the RS decoder has received $n$ symbols or erasures from the EI device, it decodes them and outputs $k$ decoded symbols with the aid of error-and-erasure RS decoding [3], [4]. The uninterferred signal corresponding to the $i$ th FSK tone at the input of the square law detector can be expressed as

$$
\begin{aligned}
r_{i}(t)= & \alpha_{s} \sqrt{2 P R_{c}} \cos \left\{2 \pi\left(f_{i}\right) t+\phi_{s}\right\}+n_{i}(t), \\
& i=1,2, \ldots, M,
\end{aligned}
$$

where $\alpha_{s}$ represents the amplitude attenuation factor due to Rayleigh fading and $\phi_{s}$ includes all the phases in the received signal due to frequency hopping, carrier modulation and MFSK modulation as well as that induced by the fading channel. Finally, $n_{i}(t)$ represents the AWGN having zero mean and double-sided power spectral density of $N_{0} / 2$.

Assuming that the first FSK tone is activated, let us denote the output of the square-law detector corresponding to the signal tone by $U_{1}$, when it is uninterferred, and by $U_{1(j)}$ when the frequency of the interfering tone coincides with that of the signal tone. Similarly, let $U_{i}$ and $U_{i(j)}$ denote the corresponding unjammed and jammed outputs of the square-law detector corresponding to the $i$ th non-signal tone. Assuming independent Rayleigh fading of the desired signal and the jamming tones, it can be shown that the outputs of the square-law detectors can be expressed as [5], [6]

$$
\begin{gathered}
U_{1(j)}=\left|\alpha_{s} \sqrt{R_{c} E_{s}} e^{j \phi_{s}}+\alpha_{j} \sqrt{E_{j}} e^{j \phi_{j}}+n_{1}\right|^{2}, \\
U_{i}=\left|n_{i}\right|^{2} \quad i=2,3, \ldots, M, \\
U_{1}=\left|\alpha_{s} \sqrt{R_{c} E_{s}} e^{j \phi_{s}}+n_{1}\right|^{2}
\end{gathered}
$$

and

$$
U_{i(j)}=\left|\alpha_{j} \sqrt{E_{j}} e^{j \phi_{j}}+n_{i}\right|^{2},
$$

where $\alpha_{j}$ and $\phi_{j}$ are the amplitude attenuation factor and the phase associated with the interference tone, respectively. It is seen in (4) to (7) that all the square-law detector outputs are central Chisquared distributed with double degrees of freedom or exponentially distributed [5]. Thus, it can be shown that the PDFs of the noisenormalized energy detector outputs can be expressed as

$$
\begin{gathered}
f_{U_{1(j)}}(y)=\frac{1}{1+\gamma_{c(j)}} \exp \left[-\frac{y}{1+\gamma_{c(j)}}\right], \quad y \geq 0, \\
f_{U_{i}}(y)=\exp (-y), \quad y \geq 0, i>1, \\
f_{U_{1}}(y)=\frac{1}{1+\gamma_{c}} \exp \left[-\frac{y}{1+\gamma_{c}}\right], \quad y \geq 0
\end{gathered}
$$

and

$$
f_{U_{i(j)}}(y)=\frac{1}{1+\gamma_{j}} \exp \left[-\frac{y}{1+\gamma_{j}}\right], \quad y \geq 0, i>1,
$$

where $\gamma_{c}=\Omega_{c} E_{s} / N_{0}, \gamma_{j}=\Omega_{j} E_{j} / N_{0}$ and $\gamma_{c(j)}=\gamma_{c}+\gamma_{j}$. Let $H_{1}$ represent the hypothesis that the transmitted symbol is correctly detected and $H_{0}$ denote the hypothesis that the transmitted symbol is incorrectly detected, when using hard decisions. It can be shown that the probability of a correct decision for the uncoded system is given by

$$
\begin{aligned}
P_{c} & =\frac{1}{M} \int_{0}^{\infty} f_{U_{1(j)}}(y)\left[\int_{0}^{y} f_{U_{i}}(x) d x\right]^{M-1} d y+\left(\frac{M-1}{M}\right) \\
& \times \int_{0}^{\infty} f_{U_{1}}(y) \int_{0}^{y} f_{U_{i(j)}}(x) d x\left[\int_{0}^{y} f_{U_{i}}(x) d x\right]^{M-2} d y \\
& =\frac{1}{M} \sum_{n=0}^{M-1}(-1)^{n}\left(\begin{array}{c}
M-1 \\
n
\end{array}\right) \frac{1}{1+n\left(1+\gamma_{c(j)}\right)} \\
& +\left(\frac{M-1}{M}\right) \sum_{n=0}^{M-2}(-1)^{n}\left(\begin{array}{c}
M-2 \\
n
\end{array}\right)\left[\frac{1}{1+n\left(1+\gamma_{c}\right)}\right. \\
& \left.-\frac{1+\gamma_{j}}{2+\gamma_{c}+\gamma_{j}+n\left(1+\gamma_{c}\right)\left(1+\gamma_{j}\right)}\right]
\end{aligned}
$$

Thus we have $P\left(H_{0}\right)=P_{c}$ and the probability of symbol error is expressed as

$$
P\left(H_{0}\right)=P_{s e}=1-P_{c} .
$$

\section{RS-CODED SYSTEM USING ERROR-AND-ERASURE DECODING}

Let us now investigate the achievable performance of the SFHMFSK system, when error-and-erasure decoding is considered. We choose three different EI schemes, each of which can be employed for inserting an erasure after energy detection on the basis of a certain test condition. Let $Y_{1}=\max \left[U_{1}, U_{2}, \ldots, U_{M}\right]$ and $Y_{2}=$ $\max _{2}\left[U_{1}, U_{2}, \ldots, U_{M}\right]$ denote the maximum and second maximum of the square-law detector outputs $U_{i}, i=1,2,3, \ldots, M$. In the context of the OTT, if $Y_{1} \leq Y_{T}$ is satisfied, where $Y_{T}$ is a preset threshold, the associated demodulated symbol should be erased. Otherwise, if we have $Y_{1}>Y_{T}$, the demodulator outputs an RS code symbol [3]. By contrast, in the context of the RTT, a pre-set threshold $\lambda_{T}$ can be invoked, in order to erase the low-reliability symbols, whenever we have $Y_{2} / Y_{1} \geq \lambda_{T}$ [1], [3]. Finally, in the context of the joint MO-RTT [3], we assume that $Y_{T}$ and $\lambda_{T}$ are two thresholds, which activate an erasure insertion, whenever we have $Y_{1} \leq Y_{T}$ and $Y_{2} / Y_{1} \geq \lambda_{T}$.

Next, we determine the expressions for the conditional PDFs of $Y_{1}$ in the context of the OTT, of $\lambda=Y_{2} / Y_{1}$ in the context of the RTT as well as the joint PDF of $Y_{1}$ and $\lambda=Y_{2} / Y_{1}$ in the context of the MO-RTT, when the SFH-MFSK system operates in Rayleigh fading in the presence of $n=1$-band MTJ. The PDF of $Y_{1}$, conditioned on the correct decision hypothesis of $H_{1}$, can be expressed as follows

$$
\begin{aligned}
& f_{Y_{1}}\left(y_{1} \mid H_{1}\right)=\frac{1}{P\left(H_{1}\right)} \frac{d}{d y_{1}}\left\{\frac{1}{M} \int_{0}^{y_{1}} f_{U_{1}(j)}(x) d x\right. \\
& \times \quad\left[\int_{0}^{x} f_{U_{i}}(y) d y\right]^{M-1}+\left(1-\frac{1}{M}\right) \int_{0}^{y_{1}} f_{U_{1}}(x) d x \\
&\left.\times \quad\left[\int_{0}^{x} f_{U_{i(j)}}(y) d y\right]\left[\int_{0}^{x} f_{U_{i}}(y) d y\right]^{M-2}\right\} \\
&=\frac{1}{P\left(H_{1}\right)} \frac{1}{M}\left[\frac{1}{1+\gamma_{c(j)}} \exp \left(\frac{-y_{1}}{1+\gamma_{c(j)}}\right)\left(1-e^{-y_{1}}\right)^{M-1}\right. \\
&+\quad(M-1) \frac{1}{1+\gamma_{c}} \exp \left(\frac{-y_{1}}{1+\gamma_{c}}\right)\left(1-e^{-y_{1}}\right)^{M-2}
\end{aligned}
$$




$$
\left.\times \quad\left[1-\exp \left(\frac{-y_{1}}{1+\gamma_{j}}\right)\right]\right] .
$$

When deriving the PDF $f_{Y_{1}}\left(y_{1} \mid H_{0}\right)$, we consider the fact that if a non-signal tone is jammed, the largest of the square-law detector outputs, i.e. $Y_{1}$, may correspond to either the jammed non-signal tone or to one of the non-signal tones unaffected by jamming. Thus, we have

$$
\begin{aligned}
& f_{Y_{1}}\left(y_{1} \mid H_{0}\right)=\frac{1}{P\left(H_{0}\right)}\left(\frac{M-1}{M}\right)\left[\left(1-e^{-y_{1}}\right)^{M-2}\right. \\
\times & {\left[1-\exp \left(\frac{-y_{1}}{1+\gamma_{c(j)}}\right)\right] e^{-y_{1}}+\left[1-\exp \left(\frac{-y_{1}}{1+\gamma_{c}}\right)\right] } \\
\times & \left\{(M-2) e^{-y_{1}}\left(1-e^{-y_{1}}\right)^{M-3}\left[1-\exp \left(\frac{-y_{1}}{1+\gamma_{j}}\right)\right]\right. \\
+ & \left.\left.\frac{1}{1+\gamma_{j}} \exp \left(\frac{-y_{1}}{1+\gamma_{j}}\right)\left(1-e^{-y_{1}}\right)^{M-2}\right\}\right] .
\end{aligned}
$$

The joint PDFs of $Y=Y_{1}$ and $\lambda=Y_{2} / Y_{1}$ conditioned on the hypothesis $H_{1}$ and $H_{0}$ can be obtained from the joint PDFs of $Y_{1}$ and $Y_{2}$ following the approach of [3], yielding

$$
\begin{aligned}
& f_{Y, \lambda}\left(y, r \mid H_{1}\right)=\left(\frac{M-1}{M}\right) \frac{y}{P\left(H_{1}\right)}\left[\frac{1}{1+\gamma_{c(j)}} e^{-y r}\right. \\
\times & \exp \left(\frac{-y}{1+\gamma_{c(j)}}\right)\left(1-e^{-y r}\right)^{M-2}+\frac{1}{1+\gamma_{c}} \exp \left(\frac{-y}{1+\gamma_{c}}\right) \\
\times \quad & \left\{(M-2) e^{-y r}\left[1-\exp \left(\frac{-y r}{1+\gamma_{j}}\right)\right]\left(1-e^{-y r}\right)^{M-3}\right. \\
+ & \left.\left.\frac{1}{1+\gamma_{j}} \exp \left(\frac{-y r}{1+\gamma_{j}}\right)\left(1-e^{-y r}\right)^{M-2}\right\}\right]
\end{aligned}
$$

and

$$
\begin{array}{ll} 
& f_{Y, \lambda}\left(y, r \mid H_{0}\right)=\left(\frac{M-1}{M}\right) y \frac{1}{P\left(H_{0}\right)}\left[e^{-y} \frac{1}{1+\gamma_{c(j)}}\right. \\
\times \quad & \exp \left(\frac{-y r}{1+\gamma_{c(j)}}\right)\left(1-e^{-y r}\right)^{M-2}+(M-2) e^{-y(r+1)} \\
\times \quad & {\left[1-\exp \left(\frac{-y r}{1+\gamma_{c(j)}}\right)\right]\left(1-e^{-y r}\right)^{M-3}+\frac{1}{1+\gamma_{c}}} \\
\times \quad & \exp \left(\frac{-y r}{1+\gamma_{c}}\right)\left\{\frac{1}{1+\gamma_{j}} \exp \left(\frac{-y}{1+\gamma_{j}}\right)\left(1-e^{-y r}\right)^{M-2}\right. \\
+\quad & \left.(M-2) e^{-y}\left[1-\exp \left(\frac{-y r}{1+\gamma_{j}}\right)\right]\left(1-e^{-y r}\right)^{M-3}\right\} \\
+\quad & (M-2)\left[1-\exp \left(\frac{-y r}{1+\gamma_{c}}\right)\right]\left\{\left(1-e^{-y r}\right)^{M-3}\right. \\
\times \quad & \frac{1}{1+\gamma_{j}} \exp \left(\frac{-y}{1+\gamma_{j}}\right) e^{-y r}+e^{-y} \frac{1}{1+\gamma_{j}} \exp \left(\frac{-y r}{1+\gamma_{j}}\right) \\
\times \quad\left(1-e^{-y r}\right)^{M-3}+(M-3) e^{-y(r+1)}\left(1-e^{-y r}\right)^{M-4} \\
\left.\left.\times \quad\left[1-\exp \left(\frac{-y r}{1+\gamma_{j}}\right)\right]\right\}\right] .
\end{array}
$$

Finally, the PDFs corresponding to the ratio $\lambda=Y_{2} / Y_{1}$ in the context of the RTT assisted EI scheme may be readily obtained from (16) and (17) by integrating them in terms of $Y$ from 0 to $\infty$, yielding

$$
\begin{aligned}
& f_{\lambda}\left(r \mid H_{1}\right)=\int_{0}^{\infty} f_{Y, \lambda}\left(y, r \mid H_{1}\right) d y=\left(\frac{M-1}{M}\right) \frac{1}{P\left(H_{1}\right)} \\
\times \quad & {\left[\sum _ { n = 0 } ^ { M - 2 } ( - 1 ) ^ { n } ( \begin{array} { c } 
{ M - 2 } \\
{ n }
\end{array} ) \left\{\frac{1+\gamma_{c(j)}}{\left[1+r(n+1)\left(1+\gamma_{c(j)}\right)\right]^{2}}\right.\right.} \\
+ & \left.\frac{\left(1+\gamma_{c}\right)\left(1+\gamma_{j}\right)}{\left[1+\gamma_{j}+r\left(1+\gamma_{c}\right)\left\{1+n\left(1+\gamma_{j}\right)\right\}\right]^{2}}\right\} \\
+ & (M-2) \sum_{n=0}^{M-3}(-1)^{n}\left(\begin{array}{c}
M-3 \\
n
\end{array}\right)\left\{\frac{1+\gamma_{c}}{\left[1+r(n+1)\left(1+\gamma_{c}\right)\right]^{2}}\right. \\
- & \left.\left.\frac{\left(1+\gamma_{c}\right)\left(1+\gamma_{j}\right)^{2}}{\left[1+\gamma_{j}+r\left(1+\gamma_{c}\right)\left\{1+(n+1)\left(1+\gamma_{j}\right)\right\}\right]^{2}}\right\}\right]
\end{aligned}
$$

and

$$
\begin{aligned}
& f_{\lambda}\left(r \mid H_{0}\right)=\int_{0}^{\infty} f_{Y, \lambda}\left(y, r \mid H_{0}\right) d y=\left(\frac{M-1}{M}\right) \frac{1}{P\left(H_{0}\right)} \\
& \times\left[\sum _ { n = 0 } ^ { M - 2 } ( - 1 ) ^ { n } ( \begin{array} { c } 
{ M - 2 } \\
{ n }
\end{array} ) \left\{\frac{1+\gamma_{c(j)}}{\left[r+(n r+1)\left(1+\gamma_{c(j)}\right)\right]^{2}}\right.\right. \\
& \left.+\frac{\left(1+\gamma_{c}\right)\left(1+\gamma_{j}\right)}{\left[1+\gamma_{c}+r\left(1+\gamma_{j}\right)\left\{1+n\left(1+\gamma_{c}\right)\right\}\right]^{2}}\right\} \\
& +(M-2) \sum_{n=0}^{M-3}(-1)^{n}\left(\begin{array}{c}
M-3 \\
n
\end{array}\right)\left\{\frac{1}{[1+r(n+1)]^{2}}\right. \\
& -\frac{\left(1+\gamma_{c(j)}\right)^{2}}{\left[r+\{1+r(n+1)\}\left(1+\gamma_{c(j)}\right)\right]^{2}} \\
& +\frac{1+\gamma_{c}}{\left[r+(r n+1)\left(1+\gamma_{c}\right)\right]^{2}}+\frac{1+\gamma_{j}}{\left[r+(r n+1)\left(1+\gamma_{j}\right)\right]^{2}} \\
& +\frac{1+\gamma_{j}}{\left[1+r(n+1)\left(1+\gamma_{j}\right)\right]^{2}} \\
& -\frac{\left(1+\gamma_{c}\right)^{2}\left(1+\gamma_{j}\right)}{\left[1+\gamma_{c}+r\left(1+\gamma_{j}\right)\left\{1+(n+1)\left(1+\gamma_{c}\right)\right\}\right]^{2}} \\
& \left.-\frac{\left(1+\gamma_{c}\right)\left(1+\gamma_{j}\right)\left(2+\gamma_{c}+\gamma_{j}\right)}{\left[(1+n r)\left(1+\gamma_{c}\right)\left(1+\gamma_{j}\right)+r\left(2+\gamma_{c}+\gamma_{j}\right)\right]^{2}}\right\} \\
& +(M-2)(M-3) \sum_{n=0}^{M-4}(-1)^{n}\left(\begin{array}{c}
M-4 \\
n
\end{array}\right)\left\{\frac{1}{[1+r(n+1)]^{2}}\right. \\
& +\frac{\left(1+\gamma_{c}\right)^{2}\left(1+\gamma_{j}\right)^{2}}{\left[\{1+r(n+1)\}\left(1+\gamma_{c}\right)\left(1+\gamma_{j}\right)+r\left(2+\gamma_{c}+\gamma_{j}\right)\right]^{2}} \\
& -\frac{\left(1+\gamma_{j}\right)^{2}}{\left[r+\{1+r(n+1)\}\left(1+\gamma_{j}\right)\right]^{2}} \\
& \left.\left.-\frac{\left(1+\gamma_{c}\right)^{2}}{\left[r+\{1+r(n+1)\}\left(1+\gamma_{c}\right)\right]^{2}}\right\}\right] \text {. }
\end{aligned}
$$

From the PDFs derived above, it is straightforward to express the probability $P_{e}$ of erasure and probability $P_{t}$ of error after erasure for a chosen threshold corresponding to a certain EI scheme. The required expressions have been given in [3]. Consequently, the probability of not decoding the codeword correctly, i.e. the codeword error probability $P_{w}$, can be expressed as [3]

$$
\begin{aligned}
P_{w} & =\sum_{i=0}^{n} \sum_{j=j_{0}(i)}^{n-i}\left(\begin{array}{c}
n \\
i
\end{array}\right)\left(\begin{array}{c}
n-i \\
j
\end{array}\right) \\
& \times\left(P_{t}\right)^{i}\left(P_{e}\right)^{j}\left(1-P_{t}-P_{e}\right)^{n-i-j},
\end{aligned}
$$


where we have $j_{0}(i)=\max \{0, n-k+1-2 i\}$. The symbol error rate after error-and-erasure RS decoding can be expressed as [8]

$$
\begin{aligned}
P_{s} & \approx \frac{1}{n} \sum_{i=0}^{n} \sum_{j=j_{0}(i)}^{n-i}(i+j)\left(\begin{array}{c}
n \\
i
\end{array}\right)\left(\begin{array}{c}
n-i \\
j
\end{array}\right) \\
& \times\left(P_{t}\right)^{i}\left(P_{e}\right)^{j}\left(1-P_{t}-P_{e}\right)^{n-i-j} .
\end{aligned}
$$

The BER of the SFH-MFSK system employing error-and-erasure RS decoding can be determined from the symbol error rate expression of (21) [5].

\section{RESULTS AND DISCUSSION}

Based on the BER derived for the RS-coded system employing one of the three EI schemes considered, we are now capable of studying the properties of the system. In Fig. 1, the probability of erasure corresponding to $\lambda_{T}=0.4$ in the context of the RTT has been shown based on both analytical and simulation results, when the SFH-MFSK system communicates in a Rayleigh fading channel and is interferrred by $n=1$-band MTJ. The results shown in Fig. 1 are for $M=2,4$ and 8 and indicate that our analysis of the probability of erasure is correct.

In Figs. 2 and 3, we evaluated the codeword error probability against a range of $Y_{T}$ and $\lambda_{T}$ values, respectively, as well as for various values of $M$ and the corresponding RS codes. We observe that for each value of $M$, an optimum threshold value exists in the context of both the OTT and the RTT, corresponding to which the minimum system codeword error probability is achieved. We also observe that the system corresponding to $M=16$ achieves the lowest codeword error probability in both figures. It is well-known that the performance of the MFSK system in the absence of interference improves as $M$ increases [5], whereas the detrimental effects of tone jamming on the performance of FH-MFSK increase as $M$ is increased [6]. Moreover, in the context of the RS-coded system, using a higher value of $M$ implies a high error correcting capability given by $t=\frac{n-k}{2}$, when the code rate is a constant [5]. Consequently, the combined effects of interference, fading, transmitted symbol energy and error correcting capability result in the observation that the system corresponding to $M=16$ achieves the best performance when using error-and-erasure RS-decoding, as portrayed in Figs. 2 and 3. In a similar fashion, it can be shown that in the context of the MO-RTT there exist optimum values of the thresholds of $\lambda_{T}$ and $Y_{T}$, which result in the best system performance, when employing error-and-erasure aided RS-decoding. In Fig. 4, we evaluated the BER of RS-coded SFH-MFSK, when it employs OTT based EI and when assuming optimum threshold values for each value of $E_{b} / N_{j}$ and $M$. The results of Fig. 4 show that error-and-erasure RS-decoding outperforms the 'error-correcting only' decoding for all values of $M$, provided that the optimum thresholds of EI are employed. However, the BER improvement of error-and-erasure decoding portrayed in Fig. 3 is noteworthy only when the jammer power is sufficiently low. Furthermore, when the signal to jamming power ratio is sufficiently high, i.e. for example when $E_{b} / N_{j}$ exceeds $20 d B$, a higher performance gain can be achieved, especially in the case of $M=32$, owing to the higher error correcting capability of the RS $(31,20)$ code used. Finally, in Fig. 5 we show our BER performance comparison for the RS-coded SFH-MFSK system, when employing the three different types of EI schemes considered. We can see that the BERs of the three types of EI schemes are hardly distinguishable from each other when $E_{b} / N_{j}$ is relatively low, i.e. for example when $E_{b} / N_{j} \leq 20 \mathrm{~dB}$. By contrast, when $E_{b} / N_{j}$ is sufficiently high, i.e. for example when

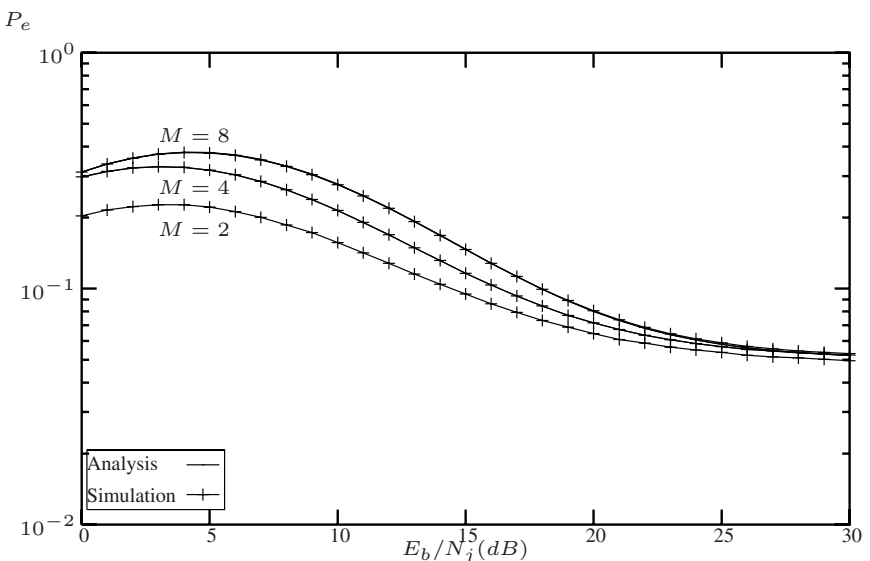

Fig. 1. Analytical and simulation results for the probability of erasure corresponding to the RTT plotted against $E_{b} / N_{j}$, when the SFH-MFSK system is subjected to Rayleigh fading and $n=1$-band MTJ and when assuming $\lambda_{T}=0.4$ and $E_{b} / N_{0}=16 d B$

$P_{w}$

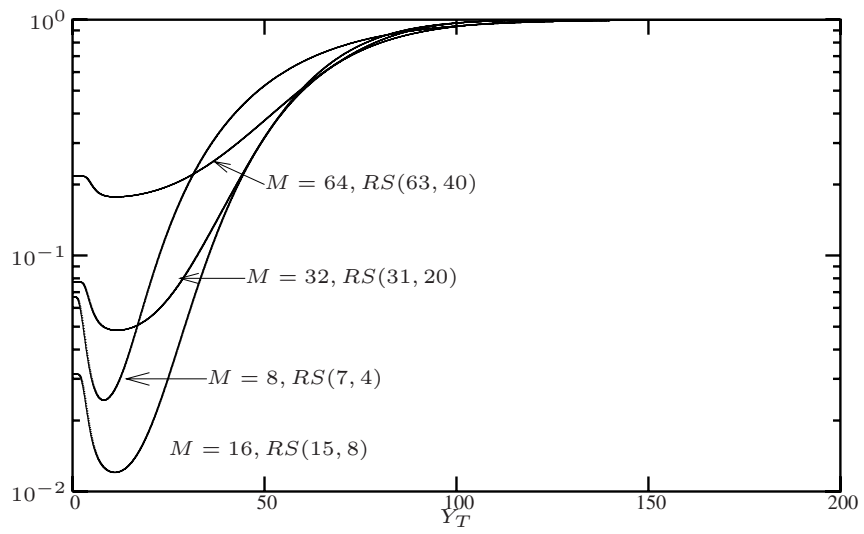

Fig. 2. Codeword error probability versus threshold $Y_{T}$ for RS-coded SFHMFSK using the OTT, when subjected to Rayleigh fading and $n=1$-band MTJ at $E_{b} / N_{j}=20 d B, E_{b} / N_{0}=16 d B$ and various values of $M$. $P w$

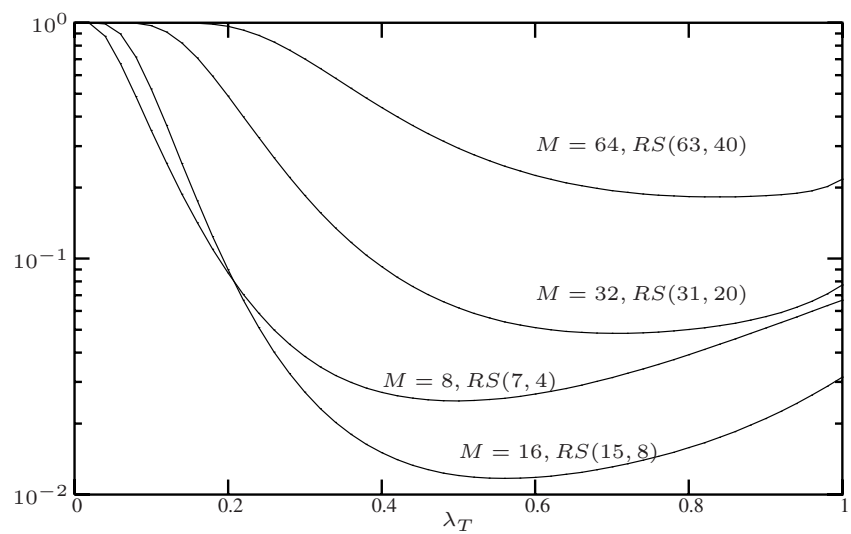

Fig. 3. Codeword error probability versus threshold $\lambda_{T}$ for RS-coded SFHMFSK using the RTT, when subjected to Rayleigh fading and $n=1$ band MTJ at $E_{b} / N_{j}=20 d B, E_{b} / N_{0}=16 d B$ and various values of $M$.

$E_{b} / N_{j} \geq 25 d B$, both the OTT and the MO-RTT outperform the RTT. It can be inferred from the definition of the EI schemes outlined in Section III that the OTT based scheme provides a technique for erasing symbols that suffer from strong fading while the RTT based 
method seeks to eliminate symbols that are rendered unreliable owing to interference. Consequently, when the jamming power is low, most errors occur due to the Rayleigh fading and the OTT based EI outperforms the RTT based scheme, as seen in Fig. 5. Finally, the MO-RTT based EI performs slightly better than both the OTT and the RTT based EI, since it makes use of the combined information based on the OTT and the RTT. However, for the system under consideration, the BER performance of the MO-RTT is close to that of the OTT. In order to show the slight differences in detail, the minimum achievable BER values of SFH 16-ary FSK have been summarised in Table I, when invoking the RTT, the OTT and the MO-RTT based EI schemes. It is noteworthy from the results seen in Table I that when $E_{b} / N_{j}$ is relatively low, i.e. 15 or $20 \mathrm{~dB}$, and $E_{b} / N_{0}$ is high, i.e. 20 or $25 \mathrm{~dB}$, the RTT outperforms the OTT. For all other values of $E_{b} / N_{j}$ and $E_{b} / N_{0}$ shown in Table I, the OTT performs better than the RTT based EI scheme. This observation implies that, when the thermal noise power is high, the OTT based scheme is more effective than the RTT based scheme.

\section{CONClusion}

In this contribution we have analyzed the BER performance of the RS-coded SFH-MFSK system using erasure insertion, when the SFHMFSK signals are transmitted over Rayleigh fading channels in the presence of $n=1$-band MTJ. We found that with the aid of errorand erasure RS decoding assisted by one of the three low complexity EI schemes considered in this contribution, the system performance may be significantly improved, provided that the jamming power is not excessively high. It was demonstrated that when the jamming power is low, the OTT outperforms the RTT. Since the MO-RTT is constituted by an amalgam of the OTT and the RTT based EI schemes, it either outperforms both the OTT and the RTT or results in a performance which matches the better of the other two. However, the corresponding performance is typically close to that of one of its counterparts. The results also showed that, in general, when the SFHMFSK system experiences tone jamming, there is an optimum value of $M$ which results in the best performance.

\section{REFERENCES}

[1] A. J. Viterbi, "A robust ratio-threshold technique to mitigate tone and partial band jamming in coded MFSK systems" Proceddings of IEEE Military Communications Conference Record, IEEE, Oct. 1982, pp. 22.4.1-22.4.5

[2] C. W. Baum and M. B. Pursley, "Bayesian methods of erasure insertion in frequency-hop communication with partial-band interference" IEEE Transactions on Communications, vol. 40, pp. 1231-1238, July 1992.

[3] L. L. Yang and L. Hanzo, "Low complexity erasure insertion in RS-coded SFH spread-spectrum communications with partial-band interference and Nakagami-m fading" IEEE Transactions on Communications, vol. 50, pp. 914-924, June. 2002.

[4] Y. T. Su and L. der Jeng, "Antijam capability analysis of RS-coded slow frequency hopped system" IEEE Transactions on Communications, vol. 48, pp. 270-281, Feb. 2000.

[5] J. G. Proakis , "Digital communications" (Mcgraw-Hill, 2001, 4th Ed.)

[6] B. K. Levitt, "FH/MFSK performance in multitone jamming" IEEE Journal on Selected Areas in Communications, vol. SAC-3, NO. 5, Sep. 1985.

[7] P. J. Crepeau, "Performance of FH/BFSK with generalized fading in worst-case partial-band gaussian interference" IEEE Transactions on Selected Areas of Communications, vol. 8, no. 5, pp. 884-886, July 1990

[8] L. L. Yang and L. Hanzo, "A residue number system based parallel communication scheme using orthogonal signaling: Part II-multipath fading channels" IEEE Transactions on Vehicular Technology, Vol. 51, NO. 6, pp. 1547-1559, November 2002.

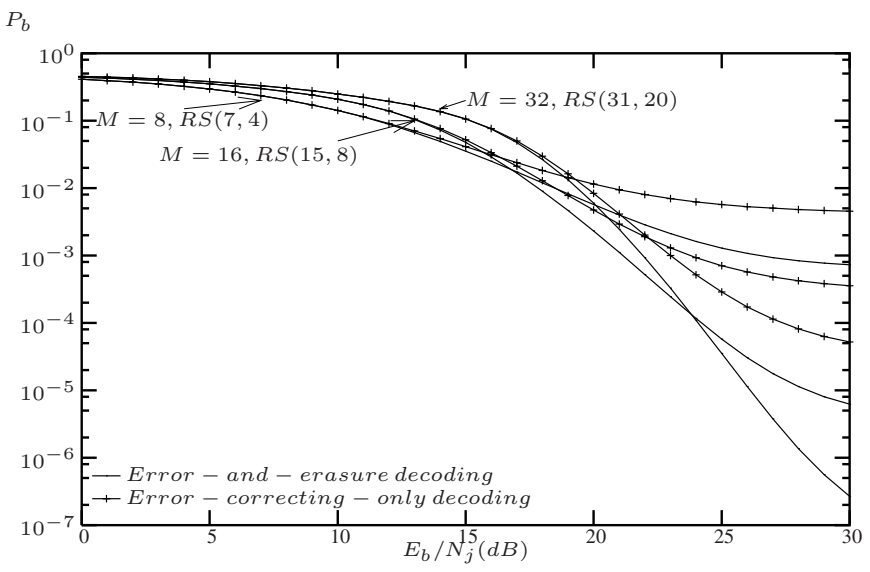

Fig. 4. BER versus $E_{b} / N_{j}$ performance of RS-coded SFH-MFSK using the OTT based EI for $E_{b} / N_{0}=16 d B$, when subjected to Rayleigh fading and $n=1$-band MTJ and assuming optimum thresholds.

$P_{b}$

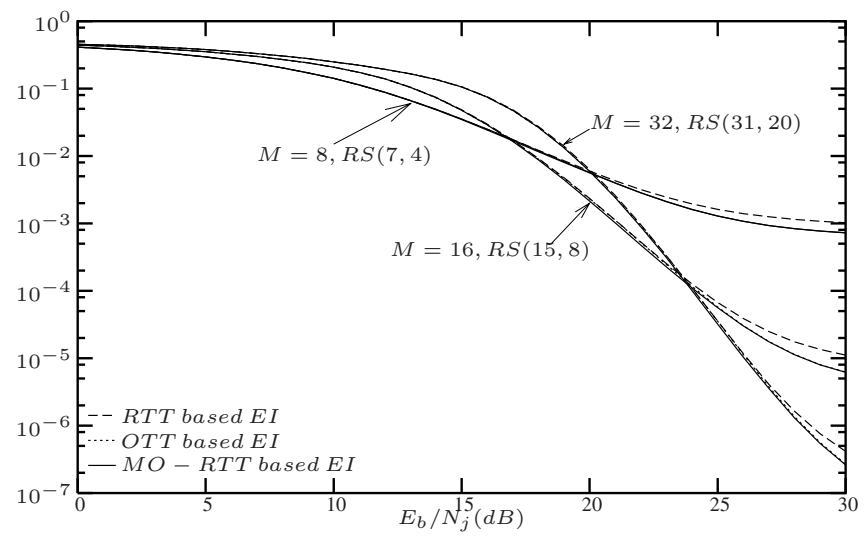

Fig. 5. Comparison of the BER versus $E_{b} / N_{j}$ performance of the RS-coded SFH-MFSK system using OTT, RTT and MO-RTT based EI for $E_{b} / N_{0}=$ $16 d B$, when subjected to Rayleigh fading and $n=1$-band MTJ and when assuming optimum thresholds.

TABLE I

COMPARISON OF BER ACHIEVED BY RS-CODED SFH 16-ARY FSK USING VARIOUS EI SCHEMES, WHEN SUBJECTED TO RAYLEIGH FADING AND $n=1$-BAND MTJ

\begin{tabular}{|c|c|c|c|c|}
\hline$E_{b} / N_{j}$ & $E_{b} / N_{0}$ & \multicolumn{3}{|c|}{ Minimum BER } \\
\cline { 3 - 5 }$(\mathrm{dB})$ & $(\mathrm{dB})$ & RTT & OTT & MO-RTT \\
\hline 15 & 5 & 0.196845 & 0.19082 & 0.19082 \\
& 10 & 0.0794641 & 0.07266 & 0.07259 \\
& 15 & 0.0503719 & 0.048873 & 0.048778 \\
& 20 & 0.0444911 & 0.045427 & 0.04432 \\
& 25 & 0.043019 & 0.04448 & 0.043019 \\
\hline 20 & 5 & 0.1682 & 0.16022 & 0.16005 \\
& 10 & 0.01828 & 0.014816 & 0.014718 \\
& 15 & 0.0028153 & 0.0026626 & 0.0025726 \\
& 20 & 0.0016041 & 0.0017065 & 0.0015004 \\
& 25 & 0.0013616 & 0.0014783 & 0.0012826 \\
\hline 25 & 5 & 0.1618 & 0.15215 & 0.15193 \\
& 10 & 0.0089072 & 0.0064891 & 0.0064363 \\
& 15 & 0.00012875 & 0.00010024 & $9.8246 \mathrm{e}-5$ \\
& 20 & $1.6447 \mathrm{e}-5$ & $1.5894 \mathrm{e}-5$ & $1.2825 \mathrm{e}-5$ \\
& 25 & $1.1437 \mathrm{e}-5$ & $9.2379 \mathrm{e}-6$ & $7.3238 \mathrm{e}-6$ \\
\hline 30 & 5 & 0.16093 & 0.15022 & 0.14999 \\
& 10 & 0.00759135 & 0.0053956 & 0.0053506 \\
& 15 & $3.55648 \mathrm{e}-5$ & $2.0125 \mathrm{e}-5$ & $1.9863 \mathrm{e}-5$ \\
& 20 & $2.11812 \mathrm{e}-7$ & $1.7064 \mathrm{e}-7$ & $1.6061 \mathrm{e}-7$ \\
& 25 & $3.63849 \mathrm{e}-8$ & $2.6517 \mathrm{e}-8$ & $1.9652 \mathrm{e}-8$ \\
\hline
\end{tabular}

